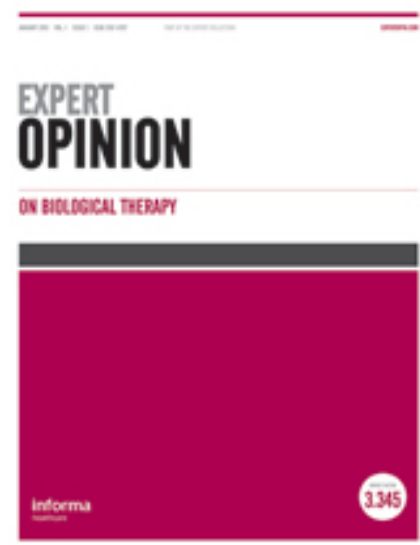

Please download and read the instructions before proceeding to the peer review

\title{
Antibody-Drug Conjugates (ADCs) delivering pyrrolobenzodiazepine (PBD) dimers for cancer therapy
}

\begin{tabular}{|r|l|}
\hline Journal: & Expert Opinion On Biological Therapy \\
\hline Manuscript ID & EOBT-2020--0072.R1 \\
\hline Manuscript Type: & Review (invited) \\
\hline Keywords: & $\begin{array}{l}\text { Antibody-drug conjugate, ADC, pyrrolobenzodiazepine dimer, PBD dimer, } \\
\text { talirine, tesirine, targeted cancer therapy }\end{array}$ \\
\hline \multicolumn{2}{|l}{} \\
\hline
\end{tabular}

\section{SCHOLARONE \\ Manuscripts}




\title{
Antibody-Drug Conjugates (ADCs) delivering pyrrolobenzodiazepine (PBD) dimers for cancer therapy
}

\begin{abstract}
Introduction:

The rationally designed pyrrolobenzodiazepine (PBD) dimers emerged around ten years ago as a new class of drug component for antibody-drug conjugates (ADC). They produce highly cytotoxic DNA cross-links, exploiting a completely different cellular target to the auristatin and maytansinoid tubulin inhibitor classes and a different mode of DNA damage to other DNA interacting warheads such as calicheamicin.
\end{abstract}

\section{Areas covered:}

The properties which make the PBD dimers suitable warheads for ADCs, and the development of the two main payload structures talirine and tesirine, are discussed. The clinical experience with the twenty PBD dimer-containing ADCs to enter the clinic is reviewed, with a focus on vadastuximab talirine and rovalpituzumab tesirine, both of which were discontinued following pivotal studies, and loncastuximab tesirine and camidanlumab tesirine which are progressing towards approval.

\section{Expert Opinion:}

Reviewing the clinical efficacy and safety data from almost forty clinical trials of PBD dimer-containing ADCs highlights the complexities and challenges of ADC early clinical development. It enables some conclusions to be made about reasons for failure and suggests strategies to optimise the future clinical development of this promising class of ADCs in a rapidly expanding field.

Keywords: ADC, Antibody-drug conjugate, pyrrolobenzodiazepine dimer, PBD dimer, talirine, targeted cancer therapy, tesirine 


\section{The introduction of PBD dimers as the drug component of antibody-drug conjugates} (ADCs)

Anthramycin was the first of the pyrrolobenzodiazepine (PBD) family of antitumour antibiotics to be discovered in the 1960s. This class of naturally occurring, and later synthetic, compounds are tricyclic systems consisting of an aromatic A-ring, a 1-4-diazepin5-one B-ring and a pyrrolidine C-ring (Figure 1A). Their mechanism of action involves sequence selective binding in the DNA minor groove of DNA and covalent binding to the exocyclic C2-amino group of guanine bases. Wholly synthetic PBD dimers, in which two PBD monomer units are joined through their C8-positions via a flexible tether, are significantly more cytotoxic due to their ability to form two covalent bonds and thereby cross-link DNA. The development of PBDs and PBD dimers as stand-alone agents has been reviewed previously $[1,2]$.

The only PBD dimer to enter the clinic, SJG-136 (SG2000, Figure 1B,C), showed potent cytotoxicity in the low nM range against human tumour cell lines in vitro [3]. Subsequently, rational structural modification of the PBD dimer pharmacophore led to enhanced DNA interstrand cross-linking ability and resultant potency, including agents with pM and in some cases sub-pM, activity in vitro [4-6]. The ability to generate molecules that displayed such exquisite potency presented significant challenges to their development as systemic agents leading to the evaluation of pro-drug strategies to increase therapeutic index [5,7]. In addition, the fact that highly potent PBD dimer molecules contain multiple potential sites of chemical attachment and can be inactivated with appropriate modification, suggested that they may have a role in other strategies aimed at targeting and releasing highly cytotoxic agents directly at a tumour site. 
The rationally designed PBD dimers therefore emerged around ten years ago as a new class of drug component for antibody-drug conjugates (ADC) to challenge the established calicheamicin, auristatin and maytansinoid classes. This has resulted in some significant successes in the clinic, but also some high-profile failures. This article will review the current status and discuss the prospects for the future of this class of ADCs.

\section{Why PBD dimers as ADC warheads?}

The mechanism of action of PBD dimer-containing ADCs is shown in Figure 1D. Initially, the main driver for the use of PBD dimers was their potency, enabling ADCs to be constructed with lower drug-antibody ratios (DAR), typically around two, than auristatin and maytansinoid-containing ADCs. This exquisite potency enabled complete regressions to be achieved in multiple pre-clinical in vivo models following just a single intravenous administration of ADC [8-11]. This increased potency also provides the ability to target low copy number antigens, which may be particularly important for the treatment of solid tumours.

The PBD dimers introduced a novel mechanism of drug action into the ADC arena, exploiting a completely different cellular target to the tubulin inhibitor classes, and a different mode of DNA damage to other DNA interacting warheads such as calicheamicin. Moreover, auristatins, maytansinoids and calicheamicins are structurally complex, whereas the PBD dimers can be made through robust and scalable synthetic routes [12]. Cell lines derived from haematological malignancies are known to be particularly sensitive to DNA damaging agents 
such as the PBD dimers, and these agents are active against solid tumour types (e.g. colon cancer) which are inherently resistant to tubulin binder drugs $[3,6]$.

An important feature of the highly cytotoxic and relatively structurally non-distorting DNA interstrand cross-links produced by the PBD dimers is their persistence in cells due to evasion of DNA repair mechanisms [3-6]. This contributes to their potency, but also to their ability to affect slowly proliferating target cells, including cancer stem, or tumour-initiating, cells, where effective eradication can ensure sustained tumour regression and prevention of recurrence. Indeed, PBD dimer SG3199 was substantially more effective at eradicating cancer stem cells in vitro than tubulin inhibitors monomethyl auristatin E (MMAE) and tubulysin, and ADCs delivering SG3199 significantly reduced the cancer stem cell population of tumours in vivo unlike tubulin inhibitor-containing ADCs [13]. Further evidence was provided by limiting dilution of cells in patient-derived xenograft (PDX) serial transplantation experiments, which indicated that the lack of tumour recurrence after PBDdimer ADC exposure resulted from the targeting of tumour-initiating cells expressing the antigen target of the ADC [14].

Following DNA interstrand cross-linking, there is a cascade of cellular events leading to cell cycle arrest and ultimately to cell death [15]. Cell cycle arrest in the G2 phase has been shown for PBD dimers [3] and subsequently for PBD dimer-containing ADCs [10]. Cells defective in homologous recombination have been shown to exhibit increased sensitivity to PBD dimers in vitro [16], and this has translated into the exquisite sensitivity of tumours harbouring such defects to PBD dimer-containing ADCs [17], potentially widening the therapeutic index considerably in this setting. In addition, inhibitors of DNA damage response can be synergistic [18]. Striking synergies have also been found between PBD 
dimer ADCs and immuno-oncology agents such as PD-1/PD-L1 antibodies in immunocompetent mouse models $[19,20]$. Immunogenic cell death produced by cytotoxic PBD dimers is thought to be contributing to the synergy which suggests rational drug combination strategies in the clinic [19].

Although P-glycoprotein expression can influence the in vitro and in vivo activity of some PBD dimers [21], it is dependent on the particular PBD structure and many potent PBD dimer warheads are not significant P-glycoprotein substrates [6,8], giving them an important advantage over other natural product-derived ADC warheads. Membrane permeability is critical in enabling bystander cell killing which may be an important determinant of efficacy in solid tumours with heterogeneous cell surface target antigen expression. ADCs with cleavable linkers delivering PBD dimers have been shown to produce efficient bystander cell killing in vitro and in vivo [10,22]. PBD dimers have a very short half-life, which can be as short as only a few minutes [6]. This ensures that the bystander effect is restricted and that systemic accumulation of free drug, which could contribute to off-target toxicity, is limited. In addition, while ADCs have a comparatively long half-life in circulation, often several days, the very short half-life of the drug should ensure that any premature release in circulation would not result in accumulation to levels that cause systemic toxicity.

In clinical stage antibody-PBD dimer conjugates, two different sites on PBD dimers have been utilised to attach linkers: C2 and N10. An example of C2-linkage is the drug-linker payload talirine (SGD-1910, Figure 2) which is conjugated to cysteine residues in an antibody. Following lysosomal cathepsin cleavage of the dipeptide valine-alanine, PBD dimer SGD-1882 is released. SGD-1882 contains phenyl rings attached to the C2 position of 
the PBD C-ring; these flat constituents can be accommodated in the DNA minor groove and the endo-exo unsaturation motif enhances cytotoxicity.

Since the N10 position in the PBD B-ring is involved in covalent binding to DNA, linker attachment at this site produces a prodrug as well as an ADC payload. As a consequence, attachment at the N10 position requires a linker that becomes completely traceless following cleavage. This is the technology employed in payload tesirine (SG3249 [23]), which releases PBD dimer SG3199 upon cathepsin cleavage of dipeptide valine-alanine and self-immolation of the PAB group (Figure 3). Tesirine also includes a discrete hydrophilic 8-unit polyethylene glycol (PEG) group which further improves solubility over the more lipophilic talirine.

To date, twenty PBD dimer-containing ADCs have entered the clinic, the vast majority of which utilise the payloads talirine or tesirine (Table 1).

\section{The rise and fall of talirine}

The use of PBD dimers as the drug component of ADCs was initially evaluated by Seattle Genetics Inc. in collaboration with Spirogen Ltd. This resulted in the development of payload talirine which first entered the clinic in 2013.

\subsection{Vadastuximab talirine}

SGN-CD33A, later to be named vadastuximab talirine, utilised the humanised anti-CD33 antibody h2HI2 engineered to contain cysteine at position 239 on the heavy chain. This allowed consistency in drug loading and low levels of aggregation compared to stochastic 
conjugation of talirine through endogenous interchain cysteines. SGN-CD33A was found to be highly active against CD33-positive human AML cell lines, regardless of MDR or p53 status [8]. It was superior to gemtuzumab ozogamicin (Mylotarg) in primary AML samples with activity demonstrated across the entire cytogenetic risk spectrum (unfavourable, intermediate and favourable). Vadastuximab talirine demonstrated complete and durable responses against subcutaneous AML xenograft models following a single dose as low as $100 \mu \mathrm{g} / \mathrm{kg}$, and potent antileukemic activity in disseminated models. In drug resistant models where gemtuzumab ozogamicin was inactive, doses up to ten-fold higher of SGN-CD33A were required. Specificity was demonstrated by lack of activity of the unconjugated antibody and equivalent dose of the free cytotoxic drug, or an unconjugated mixture of the two [8].

Based on the encouraging pre-clinical activity, a phase 1, first-in-human dose escalation study of vadastuximab talirine was conducted as monotherapy in patients with CD33-positive relapsed and refractory AML [24]. Robust, single agent antileukaemic activity was observed with minimal non-haematological toxicity. Dose-dependent, rapid elimination of ADC from the circulation was consistent with target-mediated sequestration. Most adverse events were consistent with myelosuppression, with non-haematological events including fatigue, nausea and diarrhoea. 30-day mortality was recorded as $8 \%$. The recommended monotherapy dose of vadastuximab talirine was $40 \mu \mathrm{g} / \mathrm{kg}$ and at this dose the complete remission $(\mathrm{CR})+$ complete remission with incomplete blood count recovery (CRi) rate was $28 \%$. Half of patients who responded achieved minimal residual disease negativity.

Pre-clinical in vitro studies indicated the potential of combining vadastuximab talirine with hypomethylating agents [25]. Specifically, AML cell lines demonstrated a small increase in CD33 expression after azacytidine or decitabine, and a dose-dependent increase in the 
incorporation of PBD dimer into DNA was observed following pre-treatment with the hypomethylating agent. An expansion cohort of the initial phase 1 study therefore tested the combination of a low dose of vadastuximab talirine $(10 \mu \mathrm{g} / \mathrm{kg})$ given on the last day of an azacytidine or decitabine infusion, in four-week cycles [26]. No dose-limiting toxicities were reported. The majority of adverse events were a result of myelosuppression with some causing treatment delays. In 53 patients (median age 75$)$ with adverse $(38 \%)$ or intermediate (62\%) cytogenetic risk, the $\mathrm{CR}$ and $\mathrm{CRi}$ rate was $70 \%$, with half of the remissions minimal residual disease negative. The combination, therefore, produced higher remission rates than historical hypomethylating agent monotherapy, but was accompanied by increased haematological toxicity in this older patient population.

On the strength of the impressive phase 1 results a global, randomised, double blinded, placebo-controlled phase 3 trial was launched in 2016 to compare the addition of vadastuximab talirine to hypomethylating agent therapy versus hypomethylating agent alone. This trial, named CASCADE, was terminated, however, in June 2017 due to a higher rate of deaths, including fatal infections, in the vadastuximab talirine-containing arm [26].

\subsection{Other talirine-containing ADCs}

Seattle Genetics have also clinically evaluated several other talirine-containing ADCs. SGNCD70A is an ADC directed to the antigen CD70, the cellular ligand of the tumour necrosis factor receptor family member CD27, expressed on tumour cells of a wide variety of both haematological and solid cancers [9]. A first-in-human study evaluated SGN-CD70A in patients with relapsed or refractory CD70-positive NHL, mantle cell lymphoma and Grade 3b follicular lymphoma [27]. The drug was initially administered on a three-week cycle at a dose 
escalation starting dose of $8 \mu \mathrm{g} / \mathrm{kg}$, but the schedule was amended to dose every six weeks due to prolonged thrombocytopenia. The maximum tolerated dose was determined to be 30 $\mu \mathrm{g} / \mathrm{kg}$ on the six-week schedule. Other common adverse events were nausea, anemia and fatigue. In twenty evaluable patients, one CR and three PRs were observed, two of which were ongoing for at least 42.9 weeks. Drug exposures were approximately dose proportional with a mean terminal half-life of 3 to 5 days.

SGN-CD70A was further evaluated in CD70-positive metastatic renal cell carcinoma [28]. The maximum tolerated dose was again determined to be $30 \mu \mathrm{g} / \mathrm{kg}$, with thrombocytopenia as the dose limiting toxicity. Of eighteen enrolled patients, one achieved a PR and thirteen achieved stable disease giving a clinical benefit rate of $78 \%$. Modest single-agent antitumour activity was therefore observed in these two cohorts of heavily pre-treated patients, which the company decided did not support further development.

Impressive pre-clinical data with three other talirine-containing ADCs, SGN-CD19B [29], SGN-CD123A [30] and SGN-CD352A [31], led to phase 1 trials being initiated in B-cell NHL, AML and multiple myeloma, respectively. All three studies were terminated at the decision of the sponsor and results have yet to be published. ABBV-176, targeting the prolactin receptor, a tumour associated antigen overexpressed by a variety of tumour cell types, entered phase 1 in 2017 but was terminated in 2018 due to safety concerns. The only talirine-containing ADC currently under clinical evaluation is serclutamab talirine (ABBV321), also developed by Abbvie, which utilises an affinity-matured humanized monoclonal antibody directed against the epidermal growth factor receptor (EGFR). A phase 1 study is evaluating the safety, pharmacokinetics, and antitumour activity of ABBV-321 in subjects with advanced solid tumors associated with overexpression of EGFR [32]. This is the third 
generation EGFR-targeted ADC developed by Abbvie, and it will be interesting to learn in this solid tumour setting how it compares clinically, both in terms of efficacy and toxicity profile, to the previous Depatux-m [33] and ABBV-221 [34], which deliver tubulin inhibitor MMAF and MMAE, respectively.

\section{The fall and rise of tesirine}

Early in the development of talirine, the limited aqueous solubility of this particular PBD dimer drug and the significant potential for aggregation during conjugation were noted [8]. This led to the development of novel PBD dimer-containing payloads which could be conjugated in either a stochastic or engineered manner in mostly aqueous buffer with minimal aggregation. The most widely studied of this next generation of PBD dimer payloads was tesirine developed by Spirogen Ltd.

\subsection{Rovalpituzumab tesirine}

Rovalpituzumab tesirine (originally named SC16LD6.5) is an ADC composed of a humanised IgG1 antibody SC16, conjugated to tesirine. SC16 targets delta-like 3 protein (DLL3), an atypical member of the Notch receptor ligand family that has been implicated in regulation of cell development and cell fate decisions, with a potential function as an oncogenic driver in high-grade neuroendocrine tumours, including small cell lung cancer (SCLC). In patient-derived xenograft (PDX) models, in vivo efficacy correlated with DLL3 expression, and the ADC effectively targeted and eradicated DLL3-expressing tumour initiating cells in SCLC and large cell neuroendocrine carcinoma PDX tumours [14]. 
A first-in-human phase 1 study of rovalpituzumab tesirine was undertaken in recurrent SCLC, or large cell neuroendocrine tumours, with progressive measurable disease previously treated with one or two chemotherapeutic regimens, including platinum-based [35]. It was administered every three or six weeks with doses ranging from 0.05 to $0.8 \mathrm{mg} / \mathrm{kg}$. Doselimiting toxicities including thrombocytopenia and liver function abnormalities were observed at the highest dose. The maximum tolerated dose was $0.4 \mathrm{mg} / \mathrm{kg}$ every three weeks and the recommended phase 2 dose and schedule was determined to be $0.3 \mathrm{mg} / \mathrm{kg}$ every six weeks. Roughly linear pharmacokinetics were observed with dose-proportional increases in exposure and a half-life of around 10-14 days.

At active doses $(0.2 \mathrm{mg} / \mathrm{kg}$ and above $), 18 \%$ of assessable patients had a confirmed objective response including $38 \%$ of patients confirmed to have 'high' DLL3 expression, defined as expression in $50 \%$ or more of tumour cells. Rovalpituzumab tesirine was therefore reported to have encouraging single agent activity with a manageable safety profile in this disease where few therapeutic options are available in the recurrent setting.

A phase 1 study was also performed in Japanese patients with similar safety and pharmacokinetic findings [36]. Patients received rovalpituzumab tesirine at 0.2 or $0.3 \mathrm{mg} / \mathrm{kg}$ on a six-week cycle with dexamethasone. Toxicity was generally manageable with no dose limiting toxicities observed. In DLL3 high patients, 17\% had confirmed partial responses and the disease control rate was $56 \%$ with a median progression free survival of 2.9 months and a median overall survival of 7.4 months. 
A phase 2 study named TRINITY was subsequently performed in third-line and beyond SCLC patients with DLL3-expressing, relapsed/refractory disease [37]. Rovalpituzumab tesirine was administered at $0.3 \mathrm{mg} / \mathrm{kg}$ every six weeks for two cycles. The most common adverse events were fatigue, photosensitivity reaction and pleural effusion. Overall response rate was $12.4 \%$. This was $14.3 \%$ in DLL3-high patients (greater than or equal to $75 \%$ of tumour cells positive for DLL3) where the median progression free survival was 3.8 months and the overall survival 5.7 months. The agent therefore had only very modest antitumour activity in this setting.

Furthermore, enrolment in a phase 3 trial of rovalpituzumab tesirine in the second-line setting (TAHOE study [38]) was terminated early due to shorter overall survival in the ADC arm compared to the control topotecan arm, and a phase 3 placebo-controlled trial evaluating rovalpituzumab tesirine as a first-line maintenance therapy for advanced SCLC (MERU[39]), demonstrated no survival benefit at interim analysis and was terminated at the recommendation of the Independent Data Monitoring Committee.

\subsection{Camidanlumab tesirine}

Hodgkin lymphoma (HL), various T- and B-cell non-Hodgkin lymphoma tumour cells and leukaemias express the interleukin 2 receptor alpha (IL2R- $\alpha$, CD25), and in the refractory setting there is evidence of maintenance of CD25 expression. In addition, adult T-cell leukaemia lymphoma and hairy cell leukaemia show nearly $100 \%$ expression of CD25 on their circulating tumour cells. ADCT-301 (camidanlumab tesirine) is an ADC composed of human IgG1 HuMax-TAC ${ }^{\mathrm{TM}}$ against CD25, stochastically conjugated to tesirine with a DAR of 2.3 [10]. The ADC binds human CD25 with picomolar affinity, and has highly potent and 
selective cytotoxicity against a panel of CD25-expressing human lymphoma cell lines, where a strong correlation between loss of viability and DNA interstrand cross-link formation is demonstrated. DNA damage persists, resulting in phosphorylation of histone H2AX, cellcycle arrest in $\mathrm{G}_{2} / \mathrm{M}$ and apoptosis. In vivo, a single dose of ADCT-301 results in dosedependent and targeted antitumour activity against both subcutaneous and disseminated CD25-positive lymphoma models [10].

Camidanlumab tesirine was evaluated in a phase 1 study in patients with histologically confirmed relapsed/refractory T- and B-cell NHL or classical HL (cHL). Results from this study have not been published, but results in Hodgkin lymphoma were presented at the $15^{\text {th }}$ International Conference on Malignant Lymphoma at Lugano, Switzerland in June 2019 [40]. A total of $77 \mathrm{cHL}$ patients were treated on a three-weekly schedule at doses from 3 to 300 $\mu \mathrm{g} / \mathrm{kg}$. The maximum tolerated dose was not reached. The most common all grade TEAEs $(>=20 \%)$ in the total $\mathrm{cHL}$ population were fatigue, maculopapular rash, pyrexia, increased GGT, ALT and AST, nausea, oedema and cough. Grade >=3 TEAEs of anaemia, GuillainBarre syndrome/radiculopathy and increased lipase were also observed in 7.8, 6.5 and 5.2\% of patients, respectively. The overall response rate in 75 patients was $70.7 \%$ ( $40 \% \mathrm{CR})$. At the recommended initial dose for phase 2 of $45 \mu \mathrm{g} / \mathrm{kg}$, the overall response rate was $86.5 \%$ $(48.6 \% \mathrm{CR})$. The response rate was high across all subgroups, indicating a robust antitumour activity across the relapsed/refractory $\mathrm{cHL}$ population.

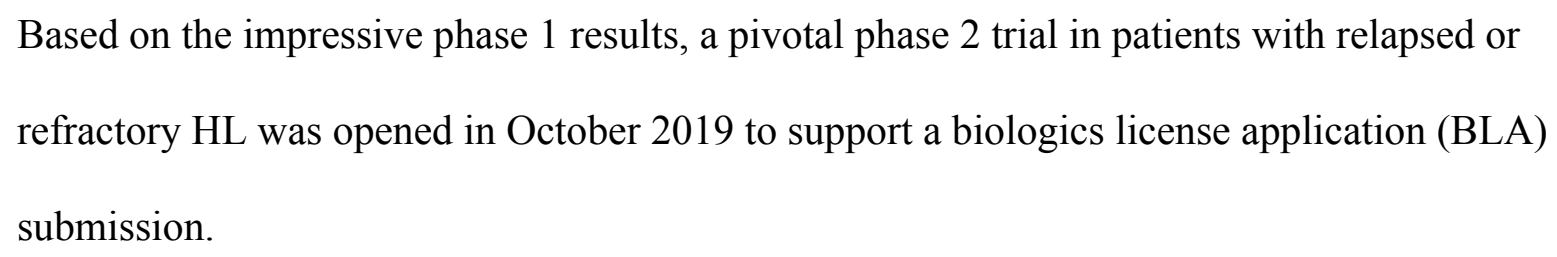
refractory HL was opened in October 2019 to support a biologics license application (BLA) submission. 
Camidanlumab tesirine also showed activity in the phase 1 study in patients with R/R NHL. Active doses with acceptable safety profiles were identified for both B-cell and T-cell lymphomas during the dose escalation phase of the study [41].

Interim data from a phase 1 study of camidanlumab tesirine in patients with $\mathrm{R} / \mathrm{R} \mathrm{CD} 25$ positive acute leukaemia were presented in 2018 [42]. No DLTs were observed up to the highest evaluated dose of $92 \mu \mathrm{g} / \mathrm{kg}$ given every three weeks, but no responses or remissions had been obtained. This study was listed as complete in 2018 and no update has been published.

In January 2019, ADC Therapeutics also dosed the first patient in a phase $1 \mathrm{~b}$ clinical trial of camidanlumab tesirine in patients with selected solid tumours that are locally advanced or metastatic [43]. CD25, the target of camidanlumab tesirine, is expressed on T regulatory cells (Tregs) that infiltrate the local tumour environment. In pre-clinical models, a single dose of the CD25-targeted ADC induced strong and durable antitumour activity against established CD25-negative solid tumours with infiltrating CD25-positive T-regs, both as monotherapy and in combination with a checkpoint inhibitor. Moreover, re-challenged mice did not develop new tumours indicating the CD25-targeted ADC was able to induce tumour-specific protective immunity $[20,44]$. Approximately 50 patients are being enrolled on this interesting proof of concept study.

\subsection{Loncastuximab tesirine}

Human CD19 antigen is a $95 \mathrm{kDa}$ type I membrane glycoprotein in the immunoglobulin superfamily, whose expression is limited to the various stages of B-cell development and 
differentiation and is maintained in the majority of B-cell malignancies, including leukemias and non-Hodgkin lymphomas of B-cell origin. Coupled with its differential and favourable expression profile, CD19 has rapid internalisation kinetics and it is not shed into the circulation, making it an ideal target for the development of ADCs. ADCT-402 (loncastuximab tesirine) is composed of the humanized IgG1 antibody RB4v1.2, directed against human CD19, stochastically conjugated to tesirine with a DAR of 2.3 [11]. It showed potent and highly targeted in vitro cytotoxicity in CD19-expressing human cell lines, and single doses of ADCT-402 resulted in dose-dependent anti-tumour activity in several subcutaneous and disseminated human tumour models in vivo, with marked superiority to comparator ADCs delivering tubulin inhibitors. Pharmacokinetic analysis in rat and cynomolgus monkey showed excellent stability and tolerability [11].

A first-in-human study was conducted to evaluate the safety and efficacy of loncastuximab tesirine in patients with relapsed/refractory B-cell NHL [45]. Patients received loncastuximab tesirine every three weeks and during dose escalation 88 patients received the drug at doses ranging from 15 to $200 \mu \mathrm{g} / \mathrm{kg}$. The most common treatment-emergent adverse events (greater than or equal to $20 \%$ patients) were haematological abnormalities, nausea, rash and dyspnoea. At doses greater than or equal to $120 \mu \mathrm{g} / \mathrm{kg}$, the overall response rate was $59.4 \%$ (40.6\% CR, 18.8\% PR). Tumour regression was observed in $69.7 \%$ of evaluable patients. After a median follow-up of 7.5 months, the overall duration of response was 5.5 months but patients achieving a CR had a durable response with duration of response not reached. The median progression free survival and overall survival (all doses) were 4.8 and 11.6 months, respectively. Data from pharmacokinetic analysis indicated that exposure increased with dose and was sustained throughout the duration of every three-week interval with no evidence of 
immunogenicity. The maximum tolerated dose was not established, but accumulating toxicity at the $200 \mu \mathrm{g} / \mathrm{kg}$ dose supported $150 \mu \mathrm{g} / \mathrm{kg}$ as the dose for expansion and phase 2 .

The majority of patients on the study were diffuse large B-cell lymphomas (DLBCL) where $54.9 \%$ (28 of 51) had a response at doses $120 \mu \mathrm{g} / \mathrm{kg}$ or above with $37.3 \% \mathrm{CR}$. Responses were also seen in nine patients with mantle cell lymphoma (44.4\% overall, $33.3 \% \mathrm{CR}$ ) where the median duration of response was 5.3 months. The median PFS was 4.8 months and the median OS was not reached. In eight patients with follicular lymphoma the overall response rate was $87.5 \%(75 \% \mathrm{CR})$. Median duration of response, progression free survival and overall survival were not reached in patients with follicular lymphoma. The US FDA have granted orphan drug designation to loncastuximab tesirine for the treatment of relapsed/refractory DLBCL and mantle cell lymphoma.

Dose expansion was undertaken at $120 \mu \mathrm{g} / \mathrm{kg}$ and $150 \mu \mathrm{g} / \mathrm{kg}$ and the resulting overall response rate for the trial at doses $120 \mu \mathrm{g} / \mathrm{kg}$ or greater was $43.3 \%(55 / 127$ patients $)$ with a CR rate of $23.6 \%$ [46]. After a median follow-up of 5.5 months, median duration of response was not reached in patients achieving a complete response.

A sub-group analysis of the phase 1 data in relapsed/refractory DLBCL by demographic and clinical characteristics revealed that older patients tolerated loncastuximab tesirine and had an encouraging overall response rate [47]. In addition, patients with three or more prior lines of therapy had a comparable overall response rate to patients with less than three lines of therapy. Patients with transformed disease also had an encouraging overall response rate. Patients with refractory DLBCL had a lower overall response rate than those with relapsed disease, but durable responses were nevertheless observed. 
A pivotal phase 2 in DLBCL, at a dose of $150 \mu \mathrm{g} / \mathrm{kg}$ every three weeks for two cycles followed by a dose of $75 \mu \mathrm{g} / \mathrm{kg}$ every three weeks was initiated. An interim futility analysis was presented at ASH in 2019 [48]. With the first 52 patients on the study, the overall response rate was $46.2 \%$, with stable disease in a further $19.2 \%$ of patients. Complete response rate was $19.2 \%$. Median duration of response was not reached for complete responders and was 5.7 months for partial responders. Seven out of ten complete responders went on to stem cell transplant or CAR-T therapy. Toxicities included GGT increase, thrombocytopaenia and neutropaenia, with effusion and skin-related treatment-related adverse events being lower than reported in the phase 1 study.

Recently, ADC Therapeutics announced positive results for the completed phase 2 trial [49]. An overall response rate of $45.5 \%$ (66/145 patients), including $20 \% \mathrm{CR}$ and $25.5 \% \mathrm{PR}$ was achieved across a broad population of relapsed or refractory patients. The data was therefore comparable to the phase 1 data at an equivalent starting dose, again with manageable toxicity. The company plan to submit a BLA to the US FDA in 2020.

Loncastuximab tesirine is also being evaluated in a phase $1 \mathrm{~b}$ trial in combination with ibrutinib in patients with R/R DLBCL or mantle cell lymphoma [50], and a phase $1 \mathrm{~b}$ trial in combination with durvalumab in patients with R/R DLBCL, mantle cell lymphoma or follicular lymphoma [51].

Loncastuximab tesirine showed only modest efficacy in B-cell acute lymphoblastic leukaemia [52]. In a phase 1 study in adults with relapsed or refractory disease, a total of 35 patients were enrolled with a median age of 55 and a median of three prior therapies. A once 
every three-week schedule demonstrated an acceptable safety profile with the most common toxicities febrile neutropenia and other haematological abnormalities and reversible liver test abnormalities. Three patients achieved complete responses at doses of 30,120 and $150 \mu \mathrm{g} / \mathrm{kg}$ every three weeks, and the maximum tolerated dose was not reached with the trial being terminated in the dose-escalation phase due to slow accrual. Therefore, although there were signals of efficacy for loncastuximab tesirine in B-ALL, the efficacy overall was less promising than in lymphomas and lower than observed for recently approved agents for relapsed or refractory B-ALL such as blinatumomab and inotuzumab ozogamycin. Despite the MTD not being reached, and therefore a recommended dose not determined, clinical activity was nevertheless observed in a heavily pre-treated patient population and efficacy could potentially be improved if it could be safely combined with chemotherapy. Preliminary evidence suggested that high levels of B-lymphocytes could limit the exposure of leukaemic cells, and B-cell depletion prior to administration of loncastuximab tesirine could possibly increase exposure and thereby efficacy.

\subsection{Other tesirine-containing ADCs}

ADC Therapeutics, either alone or in collaboration with MedImmune/AstraZeneca, have progressed several other tesirine-containing ADCs into the clinic. These include MEDI3726 (ADCT-401) for the treatment of prostate cancer. MEDI3726 is an ADC consisting of an engineered version of the anti-PSMA antibody J591 site-specifically conjugated to tesirine [53]. MEDI3726 demonstrated durable antitumour activity in PSMA-positive human prostate cancer PDX models and entered phase 1 in 2017. The study was listed as completed in 2019. 
Another tesirine-containing ADC developed by ADC Therapeutics is ADCT-502, which is comprised of the humanised antibody trastuzumab directed against HER2 site-specifically conjugated to tesirine [54]. A phase 1 clinical trial in patients with advanced sold tumours with HER2 expression was terminated early due to safety concerns. During the dose escalation phase "the necessary efficacy at tolerated doses required for patient benefit" was not achieved [55]. This was suggested to be due to the extensive expression of HER2 in pulmonary tissue. Fluid retention and pulmonary oedema are known side effects of the PBD dimers.

ADCT-602 consists of an engineered version of the humanised anti-CD22 IgG1 epratuzumab, site-specifically conjugated to tesirine with a DAR of 1.7 [56]. The first patient in a phase 1/2 clinical trial of ADCT-602 in relapsed or refractory B-cell ALL was dosed in 2018 [57]. To date, no data have been reported.

MEDI2228, a B-cell maturation antigen (BCMA)-targeted ADC entered the clinic in 2018 in a phase 1 trial of multiple myeloma. It consists of antibody BCMA-Ab1 site-specifically conjugated to tesirine and was shown in pre-clinical studies to be cytotoxic to both multiple myeloma and myeloma progenitor cells, maintaining its activity in the presence of clinically relevant levels of soluble BCMA [58]. It will be of interest to determine how this ADC compares clinically to the more advanced belantamab mafodotin developed by GSK, which targets the same antigen but delivers the tubulin inhibitor auristatin $\mathrm{F}[59,60]$

The most recent PBD dimer-containing ADC to enter the clinic is TR1801-ADC developed by Tanabe Research Laboratories. A non-agnostic cMet antibody site-specifically conjugated 
to tesirine was shown to have high activity in a range of patient-derived xenograft models of solid tumours [61].

\title{
5. Other PBD dimer-containing ADCs
}

\begin{abstract}
ADCT-601 is an ADC which utilises a humanised IgG1 antibody against human AXL, a member of the Tyro-3 Axl and Mer (TAM) family of receptor kinases over-expressed in several cancers. Overexpression is linked to metastasis, poor survival and drug resistance. In a departure from tesirine, this ADC delivers the same PBD dimer (SG3199), but in this case it is site-specifically conjugated using GlycoConnect ${ }^{\mathrm{TM}}$ technology to a valine-alanine linker containing HydraSpace ${ }^{\mathrm{TM}}$ [62]. GlycoConnect ${ }^{\mathrm{TM}}$ is a chemoenzymatic strategy that allows attachment of the payload at a specific position, and is reported to deliver ADCs with an enhanced therapeutic index compared with ADCs manufactured by random conjugation processes [63], which can be further enhanced by the use of the spacer technology HydraSpace ${ }^{\mathrm{TM}}[64]$.
\end{abstract}

A phase 1 study of ADCT-601 was initiated in 2018 in relapsed or refractory solid tumours. Interim data were presented in 2019 showing an acceptable safety profile during initial dose escalation $(50-150 \mu \mathrm{g} / \mathrm{kg})$, with preliminary evidence of activity in the ten patients with response evaluation (one unconfirmed PR, five SD) [65].

Other ADCs to reach the clinic, in which the nature of the PBD dimer is unknown, included MEDI7247 targeting ASCTII in both haematological and solid tumours [66], SC-002 targeting DLL3 in lung cancer, SC-003 targeting DPEP3 in ovarian cancer and DHES0815A targeting HER2 positive breast cancer (Table 1). 


\section{Conclusion}

The last ten years has seen a decade of discovery, development, delivery, and some disappointment in the field of PBD dimer-containing ADCs. Impressive pre-clinical data has resulted in twenty different ADCs entering the clinic, most containing the payload talirine or tesirine. Almost forty clinical trials have been completed or are currently ongoing (Table 1). Some high-profile failures have had a negative impact on the class, but recent studies showing significant activity with manageable toxicity with, for example, loncastuximab tesirine and camidanmulab tesirine, suggest that the first approval could be just around the corner.

\section{Expert Opinion}

An understanding of optimal antibody properties, linker stability and drug potency have all been key in the recent surge in activity in the ADC field. ADCs are complex, multicomponent structures and many factors can contribute to failure in the clinic. The antibody component needs to have a favourable pharmacokinetic profile which is not significantly altered following payload conjugation. Cleavable linkers need to be very stable in circulation but effectively cleaved to release drug in the appropriate target cell compartment. Truly tumour specific antigens are rare, and many antigens only show a degree of tumour selectivity. When using a highly potent warhead, such as a PBD dimer, it is becoming increasingly clear that clinical utility may depend not only on the level of expression of the target antigen on normal cells, but also its relative functional importance on key organs. 
This latter point is exemplified by the clinical experience with several tesirine-containing ADCs. In the case of loncastuximab tesirine, the target antigen CD19, in addition to being expressed on the majority of malignant B-cells, is widely expressed on normal B-cells. The depletion of these cells is clearly tolerated following ADC treatment, as is the case in other CD19-targeted therapies such as CAR-T or blinatumomab. In contrast, the expression of HER2 on critical pulmonary tissue is the likely reason for the failure of ADCT-502. Interestingly, in the case of camidanlumab tesirine, the expression of the target antigen CD25 on Tregs is being directly exploited as a non-tumour target, in addition to the highly encouraging targeting of CD25-expressing malignancies.

Despite impressive pre-clinical and early-phase clinical data with vadustuximab talirine in AML, the phase 3 CASCADE trial was terminated early due to toxicity. This highlights the difficulties inherent in translating promising phase 1 results to much larger studies, in this case of an older population of HMA-eligible patients with AML who are more susceptible to toxicity and where many develop potentially fatal cytopenias. The toxicity profile was markedly different to the non-target hepatotoxicity and veno-occlusive disease caused by the instability of Mylotarg. Expression of CD33 on normal haemopoietic precursor cells likely contributed to target-related myelosuppression. Further efforts to identify a dose regimen and cycle interval that maintains remission while minimising myelosuppression were warranted. The chequered history of gemtuzumab ozogamicin (Mylotarg), targeting the same CD33 antigen on AML, is a good example where changes to dose and schedule can revive a failed agent resulting ultimately in clinical and commercial success [67]. 
The phase 2 study TRINITY showed that rovalpituzumab tesirine had only very modest activity in third-line and beyond SCLC patients with DLL3-expressing, relapsed/refractory disease. Although the target antigen DLL3 is highly tumour selective, it is, however, a remarkably low-abundance protein on the surface of tumour cells, having in the order of only 10,000 molecules/cell when expressed [68]. This is significantly lower than other targets for ADCs such as HER2, which can express in the order of $10^{6}$ antigens per cell. The results of TRINITY did compare favourably to a retrospective multicentre study involving third-line SCLC patients treated with chemotherapy [69], and with a phase 2 study of the Trop-2 targeted ADC sacituzumab govitecan [70] which was concluded to have an effective therapeutic profile in this disease setting where treatment options are limited. Tumour selectivity may not, therefore, be sufficient for an ADC delivering a highly potent drug if the level of antigen expression is too low.

As the results of more clinical studies are published, several recurring toxicities have emerged which could be considered class effects of PBD dimer-containing ADCs. These include edema or effusion, altered liver function tests, skin-related events, myelosuppression and fatigue [71]. Many of these PBD dimer related toxicities were observed during the early phase clinical trials of standalone dimer SJG-136 [72,73] and can largely be managed clinically with appropriate scheduling and co-administration of, for example, steroids and/or diuretics. Myelosuppression is a common toxicity associated with the primary PBD dimer mode of action of DNA alkylation/cross-linking. Although dose limiting toxicities observed in animals and humans are generally related to the drug, other toxicities, including some immune-mediated events, have been observed in the clinic, often at low incidence. With increased clinical experience, an understanding of the extent to which inter-patient variability, genetic profile, and prior therapy influence tolerability, and the development of 
robust predictive biomarkers, will be crucial. Clinical experience has also indicated the importance of optimising dose scheduling (including dose reduction in later cycles) to reduce the potential for delayed toxcicity.

PBD dimer-containing ADCs have shown remarkable efficacy in pre-clinical models and this has translated into responses in the clinic in several settings. The first-in-human dose selection based on pre-clinical animal toxicity studies has generally resulted in an acceptable balance of safety and efficient dose escalation in phase 1 studies. It is interesting to note that in several studies, responses have been observed in some patients at very low starting doses $[24,45]$. Understanding why some patients are particularly sensitive to PBD dimer-containing ADCs will be important to enable future patient stratification based on predictive biomarkers. In this regard, markers for defective homologous recombination such as loss of BRCA1/2, and specific membrane transporters such as ATP-binding cassette $(\mathrm{ABC})$ drug transporters ABCG2 and ABCC2 [74], may play a role. Despite showing significant single agent activity in a number of clinical indications, the long-term development of PBD dimer-containing ADCs will depend on finding optimal combination schedules, not only with standard of care, but also with more novel agents including immunomodulatory agents, DNA damage response modulators or specific membrane transporter inhibitors.

It has been proposed that the formation and persistence of highly potent DNA interstrand cross-links produced by the PBD dimers cause significant systemic toxicities in patients and thereby limit the therapeutic index that can be achieved [75]. This has led to the development of DNA covalent-binding payloads that can only mono-alkylate rather than cross-link DNA such as the indolinobenzodiazepines (IGNs) produced by ImmunoGen Inc [76] and the pyridinobenzodiazepines (PDDs) produced by Femtogenics Ltd [77]. ADCs such as 
IMGN779 targeting CD33 [78], and IMGN632 targeting CD123 [79], showed promising preclinical activity and safety profiles suggesting a potential for increased therapeutic index in the clinic. However, a phase 1 trial of IMGN779 in AML was completed in 2019 and ImmunoGen discontinued development. Interim data was presented in 2018 where very limited efficacy was observed at the doses examined [80]. This was considerably inferior to what was observed for the PBD dimer-containing vadastuximab talirine which also targets CD33, suggesting that any reduction in toxicity may be at the expense of reduced efficacy. Recently, more encouraging data was presented for IMGN632 where a 40\% ORR rate in relapsed and refractory de novo AML patients treated at a recommended phase 2 dose was observed with manageable toxicity [81]. Since the trial disease indication of AML was the same for both IMGN779 and IMGN632, which, although targeting different antigens, both deliver the same IGN drug, this highlights the complexities and unpredictability of ADC early clinical development.

Additional strategies can be employed to reduce the possibility of off-target toxicities. These include the use of more stable likers such as the copper-free click strategies being employed by ADC Therapeutics in its most recent clinical stage ADC, ADCT-601. It will be interesting to see if the increase in therapeutic index suggested by this approach will be translated into the clinic. Concern over the potency of the PBD dimers has also led to the suggestion that lower potency drugs may be required, particularly when there is a significant level of antigen expression on critical normal cells, such as HER2 in pulmonary tissue. Lower potency drugs may necessitate an ADC with an increased DAR, and the utility of this approach has been effectively demonstrated by Daiichi Sankyo with their ADC trastuzumab deruxtecan [82] targeting a topoisomerase 1 inhibitor with a DAR of 8 gaining accelerated approval in December 2019. This approach does not, however, necessitate the move away from PBD 
dimers as the class of drug, since the flexibility of the platform allows modulation of the cytotoxic potency over several logs. Alternative approaches with the potential to increase therapeutic index include PBD dimer warheads with dual $\beta$-glucuronide and dipeptide triggers [83].

The early phase clinical data with vadastuximab talirine and the ongoing impressive clinical response data with camidanlumab tesirine and loncastuximab tesirine suggest that the therapeutic utility of PBD dimer-based ADCs lies solely in the haematological setting. Indeed, it is well documented that cells derived from haematological cancers are more inherently sensitive to PBD dimers [3,6]. This, coupled with the fact that these cancers may be more accessible to an ADC, and may express target antigens at higher levels and with greater homogeneity than many solid tumours, may contribute to this activity and the ability to achieve a satisfactory therapeutic index in some indications. Nevertheless, responses in a solid tumour setting have been observed $[28,35,36,65]$. The ability of PBD dimers to effectively kill cancer stem cells, maintain activity in multidrug-resistant cells, take advantage of deficiencies of homologous recombination in many tumour types, elicit an efficient local bystander effect and promote immunogenic cell death should all be exploitable in a solid tumour setting with an appropriately stable ADC, targeting a relatively tumour selective and abundantly expressed target antigen.

Following the announcement of positive results in the pivotal phase 2 study of loncastuximab tesirine, the submission of the first BLA for accelerated approval of a PBD-containing ADC is expected in 2020. This will meet a significant unmet need for novel therapies to treat relapsed or refractory non-Hodgkin lymphoma patients. Further submissions may follow, most likely for camidanlumab tesirine in relapsed or refractory Hodgkin lymphoma if the 
impressive response rates in phase 1 are replicated in the pivotal phase 2 . The next decade may well see PBD dimers competing with the more established ADC warheads as the numbers of approved ADCs for cancer therapy increases.

\section{Article highlights box}

- Pyrrolobenzodiazepine (PBD) dimers are highly potent DNA cross-linking agents being evaluated clinically as the drug component of antibody-drug conjugates (ADCs)

- Two different PBD dimer drug linker payloads, talirine and tesirine, have been developed independently and evaluated clinically

- To date, twenty PBD dimer-containing ADCs have entered the clinic

- Vadastuximab talirine and rovalpituzumab tesirine have progressed to pivotal studies but were subsequently discontinued

- Loncastuximab tesirine and camidanlumab tesirine are promising agents in the treatment of relapsed or refractory non-Hodgkin lymphoma and Hodgkin lymphoma, respectively

- With increasing clinical experience, strategies to optimise further clinical development of ADCs delivering PBD dimers are emerging

\section{Bibliography}

1. Hartley JA. The development of pyrrolobenzodiazepines as antitumor agents. Expert Opinion on Investigational Drugs 2011;20:733-44 
2. Mantaj J, Jackson PJ, Rahman KM, Thurston DE. From anthramycin to pyrrolobenzodiazepine (PBD)-containing antibody drug-conjugates (ADCs). Agnew Chem Int Ed Engl $2017 ; 56: 462-88$

3. Hartley JA, Spanswick VJ, Brooks N, et al. SJG-136 (NSC 694501) a novel rationally designed DNA minor groove interstrand crosslinking agent with potent and broad spectrum antitumor activity. Part 1: Cellular pharmacology, in vitro and initial in vivo antitumor activity. Cancer Research 2004;64:6693-6699

4. Hartley JA, Hamaguchi A, Suggitt M, et al. DNA interstrand cross-linking and in vivo antitumour activity of the extended pyrrolo[2,1-c][1,4]benzodiazepine dimer SG2057. Investigational New Drugs 2012;30:950-958

5. Hartley JA, Hamaguchi A, Coffils M, et al. SG2285, a novel C2-aryl-substituted pyrrolobenzodiazepine dimer pro-drug that cross-links DNA and exerts highly potent antitumor activity. Cancer Res 2010;70:6849-58

6. Hartley JA, Flynn MJ, Bingham JP, et al. Pre-clinical pharmacology and mechanism of action of SG3199, the pyrrolobenzodiazepine (PBD) dimer warhead component of antibody-drug conjugate (ADC) payload tesirine. Scientific Reports 2018;8;10479

7. Masterson LA, Spanswick VJ, Hartley JA, et al. Synthesis and biological evaluation of novel pyrrolo[2,1-c][1,4]benzodiazepine prodrugs for use in antibody-directed prodrug therapy. Bioorg Med Chem Lett 2006;16:252-6 
8. Kung Sutherland MS, Walter RB, Jeffrey SC, et al SGN-CD33A: a novel CD33-targeting antibody drug conjugate using a pyrrolobenzodiazepine dimer is active in models of drugresistant AML. Blood 2013;122(8):1455-63

9. Jeffrey SC, Burke PJ, Lyon RP, et al. A potent anti-CD70 antibody-drug conjugate combining a dimeric pyrrolobenzodiazepine drug with site-specific conjugation. Bioconjugate Chemistry 2013;24:1256-63

10. Flynn MJ, Zammarchi F, Tyrer PC, et al.ADCT-301, a pyrrolobenzodiazepine (PBD) dimer-containing antibody drug conjugate (ADC) targeting CD25-expressing hematological malignancies. Molecular Cancer Therapeutics. 2016;15:2709-21

11. Zammarchi F, Corbett S, Adams L, et al. ADCT-402, a pyrrolobenzodiazepine dimercontaining antibody-drug conjugate targeting CD-19 expressing malignancies. Blood 2018;131:1094-1105

12. Tiberghien AC, Howard PW, Goundry WRF et al. An alternative focus for route design for the synthesis of antibody-drug conjugate payloads. J Org Chem 2019;84:4830-36

13. Harper J, Lloyd C, Dimansi N, et al. Preclinical evaluation of MEDI0641, a pyrrolobenzodiazepine-conjugated antibody-drug conjugated targeting 5T4. Mol Cancer Ther 2017; $16: 15765-87$

14. Saunders LR, Bankovich AJ, Anderson WC, et al A DLL3-targetted antibody-drug conjugate eradicates high-grade pulmonary neuroendocrine tumor-initiating cells in vivo. Science Translational Medicine 2015;7(302):302ra136 
15. Osawa T, Davies D, Hartley JA. Mechanism of cell death resulting from DNA interstrand cross-linking in mammalian cells. Cell Death and Disease 2011;2:e187

16. Clingen PH, De Silva, IU, McHugh PJ, et al. The XPF-ERCC1 endonuclease and homologous recombination contribute to the repair of minor groove DNA interstrand crosslinks in mammalian cells produced by the pyrrolo[2,1-c] $[1,4]$ benzodiazepine dimer SJG-136. Nucleic Acids Res 2005; 33:3283-3291

17. Zhong H, Chen $\mathrm{C}$, Tammali R, et al. Improved therapeutic window in BRCA-mutant tumors with antibody-linked pyrrolobenzodiazepine dimers with and without PARP inhibition. Mol Cancer Ther 2019;18:89-99

18. Xing L, Lin L, Yu T, et al. A novel BCMA PBD-ADC with ATM/ATR/WEE1 inhibitors or bortezomib induce synergistic lethality in multiple myeloma. Leukemia 2020; doi: 10.1038/s41375-020-0745-9. [Epub ahead of print]

19. Rios-Doria J, Harper J, Rothstein R, et al. Antibody-drug conjugates bearing pyrrolobenzodiazepine or tubulysin payloads and immunomodulatory and synergize with multiple immunotherapies. Cancer Res 2017;77:2686-98

20. Zammarchi F, Havenith K, Bertelli F, et al. A CD25-targeted pyrrolobenzodiazepine dimer-based antibody-drug conjugate shows potent anti-tumor activity in pre-clinical models of solid tumors either alone or in combination with a PD-1 inhibitor. J Immunother Cancer 2018;6 (Suppl 1);P11 
21. Guichard SM, Macpherson JS, Thurston DE, Jodrell DI. Influence of P-glycoprotein expression on in vitro cytotoxicity and in vivo antitumour activity of the novel pyrrolobenzodiazepine dimer SJG-136. European Journal of Cancer 2005;41:1811-18

22. Li F, Emmerton KK, Jonas M, et al. Intracellular released payload influences potency and bystander-killing effects of antibody-drug conjugates in preclinical models. Cancer Res 2016;76:2710-9

23. Tiberghien AC, Levy J-N, Masterson LAS, et al. Design and synthesis of tesirine, a clinical antibody-drug conjugate pyrrolobenzodiazepine dimer payload. ACS Medicinal Chem Lett 2016; 7:983-7

24. Stein EM, Walter RB, Erba HP, et al. A phase I trial of vadastuximab talirine as monotherapy in patients with CD33-positive acute myeloid leukemia. Blood $2018 ; 131: 387-96$

25. Sutherland MSK, Yu C, O’Day C, et al. SGN-CD33A in combination with hypomethylating agents is highly efficacious in preclinical models of AML. Blood. $2015 ; 126(23): 3785$

26. Fathi AT, Erba HP, Lancet JE, et al. A phase I trial of vadastuximab talirine combined with hypomethylating agents in patients with CD33-positivge AML. Blood $2018 ; 132: 1125-33$ 
27. Phillips T, Barr PM, Park SI et al. A phase I trial of SGN-CD70A in patients with CD70positive diffuse large B cell lymphoma and mantle cell lymphoma. Invest New Drugs $2019 ; 37: 297-306$

28. Pal SK, Forero-Torres A, Thompson JA, et al. A phase I trial of SGN-CD70A in patients with CD70-positive, metastatic renal cell carcinoma. Cancer 2019;125:1124-32

29. Ryan MC, Palanca-Wessels MC, Schimpf B, et al. Therapeutic potential of SGN-CD19B, a PBD-based anti-CD19 drug conjugate, for treatment of B-cell malignancies. Blood 2017;130:2018-26

30. Li F, Sutherland MK, Yu C, et al. Characterization of SGN-CD123A, a potent CD123directed antibody-drug conjugate for acute myeloid leukemia. Mol Cancer Ther $2018 ; 17: 554-64$

31. Lewis T, Olson DJ, Gordon KA, et al. SGN-CD352A: A novel humanized anti-CD352 antibody-drug conjugate for the treatment of multiple myeloma. Cancer Res 2016;76(14 Suppl):1195

32. ClinicalTrials.gov Identifier:NCT03234712

33. Lassman AB, van den Bent MJ, Gan HK, et al. Safety and efficacy of depatuxizumab mafodotin + temozolomide in patients with EGFR-amplified, recurrent glioblastoma: results from an international phase I multicenter trial. Neuro Oncol 2019;21(1):106-14. 
34. Calvo E, Cleary JM, Moreno V, et al. Preliminary results from a phase 1 study of the antibody-drug conjugate ABBV-221 in patients with solid tumors likely to express EGFR. Journal of Clinical Oncology 2017;35.

35. Rudin CM, Pietanza MC, Bauer TM, et al. Rovalpituzumab tesirine, a DLL3-targeted antibody-drug conjugate, in recurrent small-cell lung cancer: a first-in-human, first-inclass, open-label, phase 1 study. Lancet Oncol 2017;18(1):42-51.

36. Udagawa H, Akamatsu H, Tanaka K, et al. Phase I safety and pharmacokinetics study of rovalpituzumab tesirine in Japanese patients with advanced, recurrent small cell lung cancer. Lung Cancer 2019;135:145-50

37. Morgenszterm D, Besse B, Grellier L, et al. Efficacy and safety of rovalpituzumab tesirine in third-line and beyond patients with DLL3-expressing, relapsed/refractory small-cell lung cancer:results from the phase II TRINITY study. Clin Cancer Res $2019 ; 25: 6958-66$

38. Komarnitsky P, Lee H, Shah M, et al. Rovalpituzumab tesirine vs topotecan in patients with advanced small cell lung cancer following $1^{\text {st }}$ line chemotherapy. J Thoracic Onc 2017;12:S1974-5

39. Komarnitsky PB, Lee H-J, Shah M, et al. A phase III study of rovalpituzumab tesirine maintenance therapy in patients with extensive disease small cell lung cancer. JClin Oncol 2017;35:Issue 15 supplement. TPS8583 
40. Collins G, Horwitz S, Hamadani M, et al. Analysis of clinical determinants driving safety and efficacy of camidanlumab tesirine (ADCT-301, Cami) in relapsed/refractory classical Hodgkin lymphoma. $15^{\text {th }}$ International Conference on Malignant Lymphoma, Lugano, Switzerland, June 2019

41. Collins GP, Horwitz SM, Davies A, et al. ADCT-301 (camidanlumab tesirine), a novel pyrrolobenzodiazepine-based CD25-targeting antibody drug conjugate, in a phase 1 study of relapsed/refractory non-Hodgkin lymphoma shows activity in T-cell lymphoma. Blood 2018;132 (suppl 1):1658

42. Goldberg AD, Tallman MS, Solh MM, et al. Results from an ongoing phase 1 study indicate ADCT-301 (camidanlumab tesirine) is well tolerated in patients with relapsed or refractory CD25-positive acute leukemia. Blood 2018;132 (Suppl 1):2662

43. ClinicalTrials.gov Identifier:NCT03621982

44. Zammarchi F, Chivers S and van Berkel P. Pre-clinical characterization of the mechanism of action of a CD25-targeted pyrrolobenzodiazepine dimer-based antibody-drug conjugate targeting regulatory T cells in solid cancers. J Immunother Cancer 2019;7 (Suppl 1);P697

45. Kahl BS, Hamadani M, Radford J, et al. A phase I study of ADCT-402 (Loncastuximab Tesirine), a novel pyrrolobenzodiazepine-based antibody-drug conjugate, in relapsed/refractory B-cell non-Hodgkin lymphoma. Clin Cancer Res 2019;25:6986-94 
46. Radford J, Kahl BS, Hamadani M, et al. Interim results from the first-in-human clinical trial of ADCT-402 (loncastuximab tesirine), a novel pyrrolobenzodiazepine-based antibody-drug conjugate, in relapsed/refractory diffuse large B-cell lymphoma. Blood 2018;132 (Suppl 1);398

47. Radford J, Khal B, Hamadani M, et al. Analysis of efficacy and safety of loncastuximab tesirine (ADCT-402) by demographic and clinical characteristics in relapsed/refractory diffuse large B-cell lymphoma. $15^{\text {th }}$ International Conference on Malignant Lymphoma, Lugano, Switzerland, June 2019

48. Carlo-Stella C, Zinzani PL, Kahl B, et al. Interim futility analysis of a phase 2 study of loncastuximab tesirine, a novel pyrrolobenzodiazepine-based antibody-drug conjugate, in patients with relapsed or refractory diffuse large B-cell lymphoma. Blood 2019;134(Suppl 1):757

49. https://adctherapeutics.com/press_release/adc-therapeutics-announces-first-patient-dosedin-phase-i-clinical-trial-of-adct-402-loncastuximab-tesirine-and-ibrutinib-in-patientswith-advanced-diffuse-large-b-cell-lymphoma-or-mantle-cell-lymp-7/

50. Depaus J, Bryan LJ, Ungar D, et al. Safety and anti-tumor activity study of loncastuximab tesirine and ibrutinib in diffuse large B-cell or mantle cell lymphoma. Blood 2019;134(Suppl):5309 
51. Moskowitz CH,Bastos-Oreiro M, Ungar D, et al. Safety and anti-tumor activity study of loncastuximab tesirine and durvalumab in diffuse large B-cell, mantle cell, or follicular lymphoma Blood 2019;134(Suppl):2807

52. Jain N, Stock W, Zeidan A, et al. Loncastuximab tesirine, an anti-CD19 antibody-drug conjugate, in relapsed/refractory B-cell acute lymphoblastic leukemia. Blood Adv $2019 ; 4: 449-57$

53. Cho S, Zammarchi F, Willikams DG, et al. Antitumor activity of MEDI3726 (ADCT-401), a pyrrolobenzodiazepine antibody-drug conjugate targeting PSMA, in pre-clinical models of prostate cancer. Mol. Cancer Ther 2018;17:2176-86

54. Zammarchi F, Chivers S, Williams DG, et al. ADCT-502, a novel pyrrolobenzodiazepine (PBD) dimer-based antibody-drug conjugate (ADC) targeting low HER2-expressing solid cancers. Eur J Cancer 2016;69 (Suppl 1):S28

55. https://adctherapeutics.com/press_release/adc-therapeutics-announces-the-termination-of-itsadct-502-program-targeting-her2-expressing-solid-tumors/

56. Zammarchi F, Corbett S, Janghra N, et al. ADCT-602 (hLL2-cys-PBD) a new site-specifically conjugated, pyrrolobenzodiazepine (PBD) dimer-based antibody-drug conjugate (ADC) targeting CD22-expressing B-cell malignancies. Blood 2016;128(Suppl):4176

57. ClinicalTrials.gov Identifier: NCT0369852 
58. Kinneer K, Flynn M, Thomas SB, et al. Preclinical assessment of an antibody-PBD conjugate that targets BCMA on multiple myeloma and myeloma progenitor cells. Leukemia 2019;33:766-71

59. Trudel S, Lendval N, Popat R, et al. Antibody-drug conjugate GSK2857916 in relapsed/refractory multiple myeloma: an update on safety and efficacy from dose expansion phase I study. Blood Cancer J 2019;9:37

60. Lonial S, Lee HC, Badros A, et al. Belantamab mafodotin for relapsed or refractory multiple myeloma (DREAMM-2): a two-arm, randomised, open-label, phase 2 study. Lancet Oncol 2020;21:207-21

61. Gymnopoulos M, Betancourt O, Blot V, et al. A highly potent cMet antibody-drug conjugate TR1801-ADC with high activity in PDX solid tumor models. Molecular Oncology 2019;

62. Zammarchi F, Havenith K, Chivers S, et al. Pre-clinical activity of ADCT-601, a novel pyrrolobenzodiazepine (PBD) dimer-based antibody-drug conjugate (ADC) targeting AXLexpressing tumors. Proc Am Assoc Cancer Res 2018;2792

63. van Geel R, Wijdeven MA, Heesbeen R, Verkade JM, Wasiel AA, van Berkel SS, et al. Chemoenzymatic Conjugation of Toxic Payloads to the Globally Conserved N-Glycan of Native mAbs Provides Homogeneous and Highly Efficacious Antibody-Drug Conjugates. Bioconjugate chemistry 2015;26(11):2233-42. 
64. Delft Fv, Janssen B, Geel Rv, Wijdeven M, Verkade J, Berkel Sv. Abstract 3815: Decomposition of parameters contributing to the improved therapeutic index of ADCs obtained by GlycoConnect $^{\mathrm{TM}}$ and HydraSpace ${ }^{\mathrm{TM}}$ Technologies. Cancer Res 2018;78(13 Supplement):3815-15.

65. Tolcher AW, Falchook GF, Bendell JC, et al. A phase 1, open label, dose escalation study to evaluate the safety, tolerability, pharmacokinetics and antitumor activity of ADCT-601 in patients with advanced solid tumors. Annals Oncol 2019:30 (Suppl 5):468

66. Pore N, Borrok M, Rebellato M, et al. Discovery and development of MEDI7247, a novel Pyrrolobenzodiazepine (PBD)-based antibody drug conjugate targeting ASCT2, for treating hematological and solid cancers. Cancer Research 2018;78 (Suppl) LB-296.

67. Egan PC, Reagan JL. The return of gemtuzumab ozogamicin: a humanised anti-CD33 monoclonal antibody-drug conjugate for the treatment of newly diagnosed acute myeloid leukemia. Onco Targets Ther 2018;11:8265-72

68. Sharma SK, Pourat J, Abdel-Atti D, et al. Non-invasive interrogation of DLL3 expression in metastatic small cell lung cancer. Cancer Res 2017;77:3931-41

69. Simos D, Saijady G, Sergi M, et al. Third-line chemotherapy in small-cell lung cancer; an international analysis. Clin Lung Cancer 2014;15:110-8 
70. Gray JE, Heist RS, Starodub AN, et al. Therapy of small cell lung cancer (SCLC) with a topoisomerase-I-inhibiting antibody-drug conjugate (ADC) targeting trop-2, sacituzumab govitecan. Clin Cancer Res 2017;23:5711-9

71. Saber H, Simpson N, Ricks TK, Leighton JK. An FDA oncology analysis of toxicities associated with PBD-containing antibody-drug conjugates. Regulatory Tox Pharmacol $2019 ; 107: 104429$

72. Hochhauser, D, Meyer T, Spanswick VJ, et al. Phase I study of sequence-selective minor groove DNA binding agent SJG-136 in patients with advanced solid tumors. Clinical Cancer Res 2009; 15:2140-2147

73. Puzanov I, Lee W, Chen AP, et al. Phase I, pharmacokinetic and pharmacodynamic study of SJG-136, a novel DNA sequence selective minor groove cross-linking agent, in advanced solid tumors. Clinical Cancer Res 2011;17:3794-802

74. Corbett S, Zammarchi F, Howard PW, et al. The role of transporters in the acquired resistance to PBD dimer-containing antibody-drug conjugates. Cancer Res 2019;79(13 Suppl):4750

75. Jackson PJM, Kay S, Pysz I, Thurston DE. Use of pyrrolobenzodiazepines and related covalent-binding DNA-interactive molecules as ADC payloads: is mechanism related to systemic toxicity? Drug Discovery Today: Technologies 2018;30:71-83 
76. Miller ML, Shizuka M, Wilhelm A, et al. A DNA-interacting payload designed to eliminate cross-linking improves the therapeutic index of antibody-drug conjugates (ADCs). Mol Cancer Ther 2018; 17:650-60

77. Veillard N, Andriollo P, Mantaj J, et al. Pyridinobenzodiazepines (PDDs): an new class of sequence-selective DNA mono-alkylating ADC payloads with low hydrophobicity. Cancer Res 2018;78(13 Suppl):736

78. Kovtun Y, Noordhuis P, Whiteman KR, et al. IMGN779, a novel CD33-targeting antibodydrug conjugate with DNA-alkylating activity, exhibits potent antitumour activity in models of AML. Mol Cancer Ther 2018;17:1271-9

79. Kovtun Y, Jones GE, Adams S, et al. A CD123-targeting antibody-drug conjugate, IMGN632, designed to eradicate AML while sparing normal bone marrow cells. Blood Adv 2018;2:84858

80. Cortes JE, DeAngelo DJ, Erba HP, et al. Maturing clinical profile of IMGN779, a nextgeneration CD33-targeting antibody-drug conjugate, in patients with relapsed or refractory acute myeloid. Leukemia. Blood 2018;132(suppl 1):26

81. Daver NG, Montesinos P, DeAngelo DJ, et al. Clinical profile of IMGN632, a novel CD123targeting antibody-drug conjugate (ADC), in patients with relapsed/refractory acute myeloid leukemia or blastic plasmacytoid dendritic cell neoplasm. Blood 2019;134(suppl 1):734 
82. Modi S, Saura C, Yamashita T, et al. Trastuzumab deruxtecan in previously treated HER-2 positive breast cancer. New Eng J Med 2010;382:610-21

83. Gregson SJ, Barrett AM, Patel N, et al. Synthesis and evaluation of pyrrolobenzodiazepine dimer antibody-drug conjugates with dual $\beta$-glucuronide and dipeptide triggers. Eur. J. Med. Chem 2019;179;591-607 


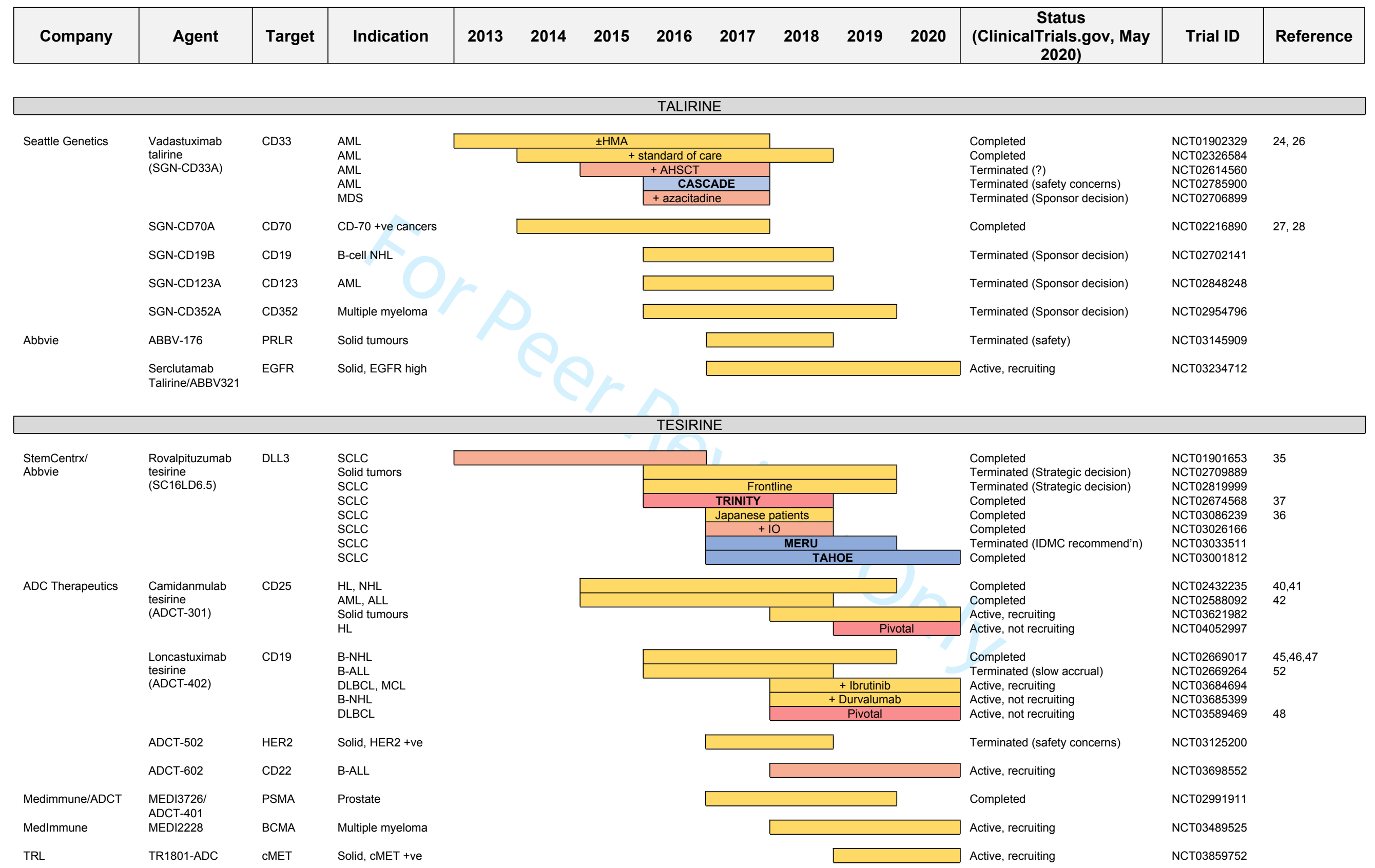




\section{Page 43 of 48}

Expert Opinion On Biological Therapy

\section{UNKNOWN/OTHER PBD PAYLOAD}

\begin{tabular}{|c|c|c|c|c|c|c|c|}
\hline & & & & UNKNOWN/OTHER PBD PAYLOAD & & & \\
\hline \multirow{2}{*}{$\begin{array}{l}\text { StemCentrx/ } \\
\text { Abbvie }\end{array}$} & SC-002 & DLL3 & SCLC, LCNEC & $\square$ & Terminated (Strategic decision) & NCT02500914 & \\
\hline & SC-003 & DPEP3 & Ovarian & \begin{tabular}{|c} 
\\
\end{tabular} & Terminated (Strategic decision) & NCT02539719 & \\
\hline \multirow[t]{2}{*}{ Medlmmune } & MEDI7247 & ASCTII & Haematological & $\square$ & Completed & NCT03106428 & \\
\hline & & & Solid tumour & $\square$ & Completed & NCT03811652 & \\
\hline ADC Therapeutics & ADCT-601 & AXL & Solid tumour & $\square$ & $\begin{array}{l}\text { Terminated (change in clinical plan } \\
\text { and drug supply) }\end{array}$ & NCT03700294 & 65 \\
\hline Genentech & DHES0815A & HER2 & HER2 +ve breast & $\square$ & Active, not recruiting & NCT03451162 & \\
\hline
\end{tabular}

Table 1. The clinical development of PBD dimer-containing ADCs 


\section{Figure legends}

Figure 1. A. The structure of the pyrrolobenzodiazepine (PBD) pharmacophore. B. The structure of PBD dimer SG2000 (SJG-136). C. Schematic representation of SG2000 binding to DNA to produce an interstrand cross-link spanning six DNA base pairs. D. The main steps in the mechanism of action of PBD dimer-containing ADCs, a) binding to antigen target on the cell surface, b) internalisation into the cell, c) trafficking to the lysosome where cleavage of the linker occurs to release the free PBD dimer, d) binding of PBD dimer in the minor groove of DNA to produce cytotoxic DNA damage, e) a cascade of cellular events leading to cell death.

Figure 2. The structure of the drug-linker payload talirine and the released PBD dimer SGD1882.

Figure 3. The structure of the drug-linker payload tesirine and the released PBD dimer SG3199. 


\section{Declaration of interest}

In addition to his academic positions, JAH was a founder scientist of Spirogen Ltd and acts as a consultant to ADC Therapeutics in which he also has an equity interest.

\section{Acknowledgements}

JAH thanks Simon Corbett, Francesca Zammarchi and Patrick van Berkel for their critical review of the manuscript prior to submission. 
A $10 \quad$ Expert Opinion On Biological Therapy

2

3

4

5

6

7

8

9

10

11

12

13

14

15

16

17

18

C

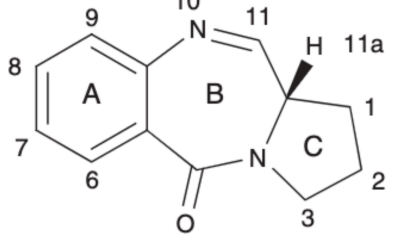

Basic PBD

Structure<smiles>C=C1C[C@H]2C=Nc3cc(OCCCOc4cc5c(cc4OC)C(=O)N4CC(=C)C[C@H]4C=N5)c(OC)cc3C(=O)N2C1</smiles>

PBD dimer

SG2000 (SJG-136)

21

22

23

24

25

26

27

28

29

30

31

32

33

34

35

36

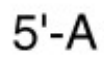

$A-G$

$\mathrm{A} \longrightarrow \mathrm{T}$

C

$T$

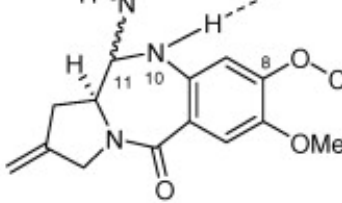

${ }^{C_{2}} C_{C_{2}}$

D

C
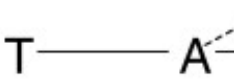

$G^{\prime}$

A

\section{7} 38

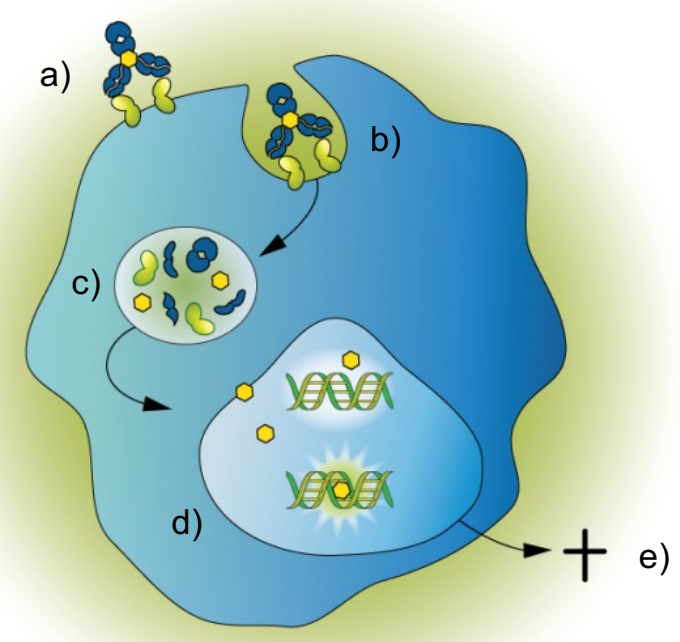




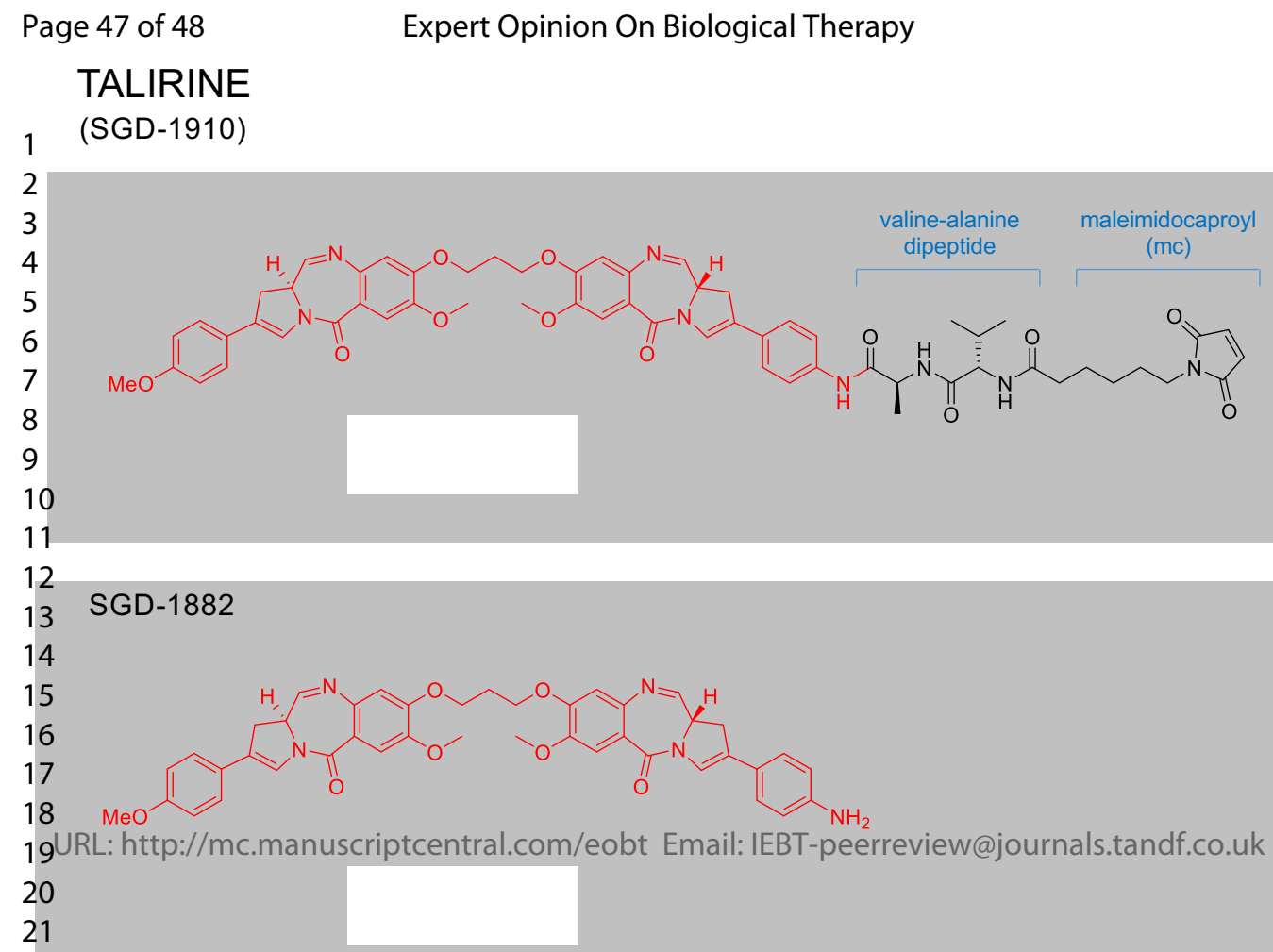
19URL: http://mc.manuscriptcentral.com/eobt Email: IEBT-peerreview@journals.tandf.co.uk 20 
(SG-3249)

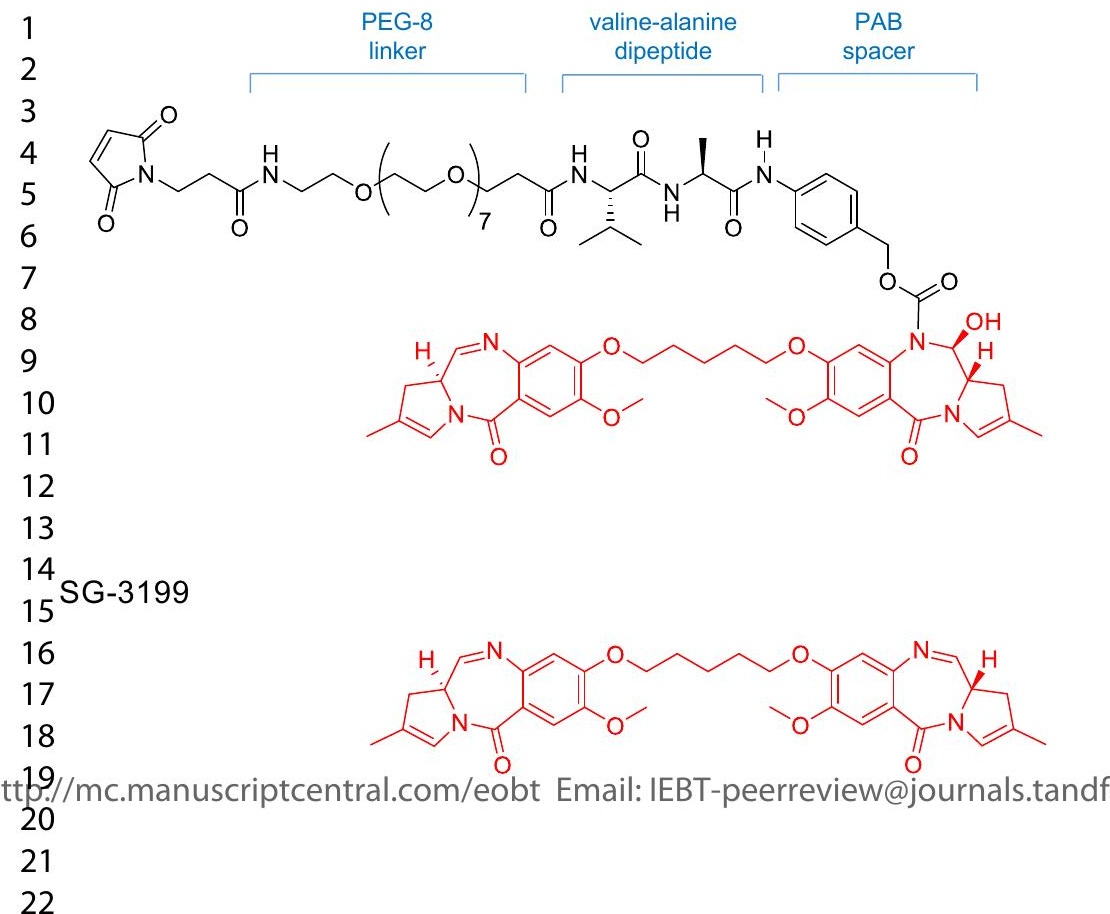

